Commun. Korean Math. Soc. 26 (2011), No. 4, pp. 649-660

http://dx.doi.org/10.4134/CKMS.2011.26.4.649

\title{
CONTINUITY OF THE ORBITAL AND LIMIT SET MAPS IN GENERAL DYNAMICAL SYSTEMS
}

\author{
Kyung Bok Lee and Jong Suh Park
}

\begin{abstract}
S. M. Saperstone and M. Nishihama [6] had showed both continuity and stability of the orbital and limit set maps, $K(x)$ and $L(x)$, where $K$ and $L$ are considered as maps from $X$ to $2^{X}$. The main purpose of this paper is to extend continuity and stability for dynamical systems to general dynamical systems.
\end{abstract}

\section{Introduction and preliminaries}

In [3], the dynamical properties is extended to general dynamical systems and differential inclusions. One of the major topics of dynamical properties is a generic property (A generic property is one that is true for a Baire set in the space of dynamical systems), which relates to the continuity of the set-valued map. In this paper, we use the terminology "motion" which was introduced in [3]. However this concept has never been used in [6].

The main purpose of this paper is to extend the following Theorem A and Theorem B for dynamical systems to general dynamical systems.

Theorem A ([6]). If $K^{+}(x)$ is compact for some $x \in X$, the following are equivalent in dynamical system.

(1) $x \in X$ is of charactorstic $O^{+}$.

(2) $K^{+}$is continuous on $X$.

(3) $K^{+}(x)$ is stable for each $x \in X$.

Theorem B ([6]). In dynamical system, a necessary and sufficient condition that the map $L^{+}$be continuous on $X$ is that $L^{+}(x)$ is eventually stable for each $x \in X$.

We prove here:

Received July 6, 2010; Revised December 6, 2010

2010 Mathematics Subject Classification. 37B40.

Key words and phrases. general dynamical systems, lower and upper semicontinuous, continuity and stability, motion and limit set, minimality and charactorstic $\mathrm{O}^{+}$.

First author is supported by the Academic Research Fund of Hoseo University in No. 20080013. 
Theorem 2.12. In general dynamical system, if $K^{+}(x)$ is compact for some $x \in X$, the following are equivalent.

(1) For each point $x \in X, x$ is of charactorstic $O^{+}$.

(2) $K^{+}$is continuous on $X$.

(3) $K^{+}(x)$ is plsitively stable on each point $x \in X$.

(4) $L^{+}(x)$ is positively stable on each point $x \in X$.

Theorem 3.12. In general dynamical system, a necessary and sufficient condition that the map $L^{+}$be continuous on $X$ is that $L^{+}(x)$ is positively eventually stable for each $x \in X$.

Continuity and stability are key notions in the stucy of the qualitive theory of dynamical systems. These widely studied by many researchers in differnt contexts. We are extended here wildy their properties on general dynamical systems to the well known result due to S. M. Saperstone and M. Nishihama $[6]$.

Generalized theory of dynamical systems [i.e., $\Phi: X \times \mathbb{R} \rightarrow X$ continuous, $\Phi(x, 0)=x$ and $\Phi(x, t+s)=\Phi(\Phi(x, t), s)]$ was introduced by Sibirsky (see chapter VI [7]).

We are many similarities when we study ordinary dynamical systems and general dynamical systems. But, there are sharp difference also while we study these properties, for instance, invariance, minimality so on. To clarify this fact it suffices to note that $q \in \Phi(p, \mathbb{R})$ does not imply the inclusion $\Phi(q, \mathbb{R}) \subset$ $\Phi(p, \mathbb{R})[7]$.

General dynamical systems, which are sometimes referred to as general control systems or set-valued dynamical systems, are used to describe multi-valued differential equations (including differential inclusions) and control systems as well as economic flows. They have been widely studied in the literature $[2,3$, 4].

We now introduced notions and definitions necessary for our works.

For a topological space $X, F(X)$ will be used to denote the set of all nonempty compact subsets of $X$.

Let $(X, d)$ be a metric space. For $A \subset X$ and $\epsilon>0$ we define

$$
B_{d}(A, \epsilon)=\{x \in X \mid d(x, A)<\epsilon\},
$$

where $d(x, A)=\inf \{d(x, a) \mid a \in A\}$.

Definition 1.1. Let $(X, d),(Y, \rho)$ be metric spaces. A set-valued map $f: X \rightarrow F(Y)$ is said to be

(1) upper semicontinuous at $x \in X$ if for any $\epsilon>0$ there exists $\delta>0$ such that $d(x, y)<\delta$ implies $f(y) \subset B_{\rho}(f(x), \epsilon)$

(2) lower semicontinuous at $x$ if for any $\epsilon>0$ there exists $\delta>0$ such that $d(x, y)<\delta$ implies $f(x) \subset B_{\rho}(f(y), \epsilon)$

(3) continuous at $x$ if $f$ is upper semicontinuous and lower semicontinuous at $x$. 
Definition 1.2. Let $(X, d),(Y, \rho)$ be metric spaces. Let $f: X \rightarrow F(Y)$ be a set-valued map. Then $f$ is called

(1) upper semicontinuous if $f$ is upper semicontinuous at every point of $X$

(2) lower semicontinuous if $f$ is lower semicontinuous at every point of $X$

(3) continuous if $f$ is upper semicontinuous and lower semicontinuous.

Definition 1.3. Let $(X, d)$ be a metric space. A set-valued map $f: X \times \mathbb{R} \rightarrow$ $F(X)$ is said to be a general dynamical system if the following axioms hold:

(1) $f(x, 0)=\{x\}$ for all $x \in X$

(2) if $s t>0$, then $f(f(x, s), t)=f(x, s+t)$ for all $x \in X$ and $s, t \in \mathbb{R}$

(3) $f$ is continuous.

\section{Continuity of the set-valued map $K^{+}$, and its positive stability}

In Sections 2 and 3, we research both continuity and stability of the setvalued maps $x \in X \rightarrow K^{+}(x) \in F(X)$ and $x \in X \rightarrow L^{+}(x) \in F(X)$, where the $K^{+}(x)$ is the positive orbit closure through $x$ and $L^{+}(x)$ is the positive limit set of $x$ in general dynamical system $f$ defined on a locally compact metric space $(X, d)$.

Theorem 2.1. $f$ is upper semicontinuous at $x \in X$ if and only if for any neighborhood $U$ of $f(x)$, there exists a neighborhood $V$ of $x$ such that $y \in V$ implies $f(y) \subset U$.

Proof. Necessity: Since $f(x)$ is compact, there exists $\epsilon>0$ such that $B(f(x), \epsilon)$ $\subset U$ for any neighborhood $U$ of $f(x)$. By upper semicontinuous of $f$ at $x$, there is $\delta>0$ such that $d(x, y)<\delta$ implys $f(y) \subset B(f(x), \epsilon) \subset U$. Set $V=B(x, \delta)$. Then $V$ is a neighborhood of $x$, and $f(y) \subset U$ for all $y \in V$.

Sufficiency: Let $x \in X$ and $\epsilon>0$. For a neighborhood $B(f(x), \epsilon)$ of $f(x)$, by assumption, there exists a neighborhood $V$ of $x$ such that $y \in V$ implies $f(y) \subset B(f(x), \epsilon)$. Thus $f$ is upper semicontinuous at $x \in X$.

Theorem 2.2. $f$ is lower semicontinuous at $x \in X$ if and only if for any open set $U$ with $U \cap f(x) \neq \emptyset$, there exists a neighborhood $V$ of $x$ such that $y \in V$ implies $U \cap f(y) \neq \emptyset$.

Proof. Necessity: Assume that $z \in U \cap f(x)$ for open set $U$ of $X$. $B(z, \epsilon) \subset U$ for some $\epsilon>0$. By the lower semicontinuity of $f$ at $x$, there is $\delta>0$ such that $d(x, y)<\delta$ implys $f(x) \subset B(f(y), \epsilon)$. Set $B(x, \delta) \equiv V$. Let $y \in V$. Since $z \in f(x) \subset B(f(y), \epsilon), d(z, w)<\epsilon$ for some $w \in f(y)$. By $w \in B(z, \epsilon) \subset U$, $U \cap f(y) \neq \emptyset$.

Sufficiency: Let $\epsilon>0 .\left\{B\left(z, \frac{\epsilon}{2}\right) \mid z \in f(x)\right\}$ is an open cover of $f(x)$. By the compactness of $f(x), f(x) \subset \bigcup_{i=1}^{k} B\left(z_{i}, \frac{\epsilon}{2}\right)$ for finitely many $z_{1}, z_{2}, \ldots$, $z_{k} \in f(x)$. For $i=1,2, \ldots, k, B\left(z_{i}, \frac{\epsilon}{2}\right)$ satisfying $B\left(z_{i}, \frac{\epsilon}{2}\right) \cap f(x) \neq \emptyset$ is an open set in $X$. Thus, $y_{i} \in V$ for some a neighborhood $V_{i}$ of $x$ implies $B\left(z_{i}, \frac{\epsilon}{2}\right) \cap f(y) \neq \emptyset$. Since $\bigcap_{i=1}^{k} V_{i}$ is a neighborhood of $x, B(x, \delta) \subset \bigcap_{i=1}^{k} V_{i}$ for some $\delta>0$. 
Let $d(x, y)<\delta$ and $w \in f(x)$. Then $w \in B\left(z_{j}, \frac{\epsilon}{2}\right)$ for some $1 \leq j \leq k$. By $y \in B(x, \delta) \subset \bigcap_{i=1}^{k} V_{i} \subset V_{j}$, we have $B\left(z_{j}, \frac{\epsilon}{2}\right) \cap f(y) \neq \emptyset$. Let $u \in B\left(z_{j}, \frac{\epsilon}{2}\right)$ $\cap f(y)$. Then $d(w, u) \leq d(w, z)+d\left(z_{j}, u\right)<\epsilon$. Therefore, $w \in B(f(y), \epsilon)$ using $d(w, f(y)) \leq d(w, u)<\epsilon$. Hence, $f(x) \subset B(f(y), \epsilon)$. Consequently, $f$ is lower semicontinuous at $x$.

For $x \in X$, the positive orbit clousre through $x$ is the set

$$
K^{+}(x)=\overline{f\left(x, \mathbb{R}^{+}\right)}
$$

and the positive limit set of $x$ is the set

$$
L^{+}(x)=\cap_{t \geq 0} \overline{f(x,[t, \infty))} .
$$

In this section, we assume that $K^{+}(x)$ is compact for all $x \in X$.

Theorem 2.3. The map $K^{+}: X \rightarrow F(X)$ is lower semicontinuous.

Proof. Let $x \in X$ and $U$ be a open set in $X$ with $U \cap K^{+}(x) \neq \emptyset$. Then $U \cap f(x, t) \neq \emptyset$ for some $t \in \mathbb{R}^{+}$. By the lower semicontinuity of $f$ at $(x, t)$, $y \in V$ for some a neighborhood $V$ of $x$ implies $U \cap f(y, t) \neq \emptyset$. Thus, $U \cap$ $f(y, t) \subset U \cap K^{+}(y)$ for $y \in V$. Therefore, $U \cap K^{+}(y) \neq \emptyset$. Hence $K^{+}$must be lower semicontinuous at $x$.

Corollary 2.4. The followings are equivalent.

(1) $K^{+}$is upper semicontinuous at $x \in X$.

(2) $K^{+}$is continuous at $x$.

For $x \in X$, the positive prolongational set of $x$ is the set

$$
D^{+}(x)=\bigcap_{U \in N_{x}} \overline{f\left(U, \mathbb{R}^{+}\right)}
$$

where $N_{x}$ is the set of all neighborhoods of $x . x \in X$ is said to be characteristic $O^{+}$if $K^{+}(x)=D^{+}(x)$.

Theorem 2.5. If $K^{+}$is upper semicontinuous at $x \in X$, then $x$ is of characteristic $\mathrm{O}^{+}$.

Proof. Assume that $x$ is not of characteristic $O^{+}$. Let $y \in D^{+}(x)-K^{+}(x)$. Then $y \notin \bar{U}$ for some neighborhood $U$ of $K^{+}(x)$. By the upper semicontinuity of $K^{+}$at $x$, there exists a neighborhood $V$ of $x$ such that $z \in V$ implies $K^{+}(z) \subset U$. Hence $y \in D^{+}(x) \subset \overline{f\left(V \times \mathbb{R}^{+}\right)} \subset \bar{U}$. We have a contradiction to the fact that $y \notin \bar{U}$. This proves that $x$ is of characteristic $O^{+}$.

Theorem 2.6. $K^{+}$is continuous at $x \in X$ if and only if $x$ is of characteristic $\mathrm{O}^{+}$.

Proof. Theorem 2.5 takes care of the "if" part. We now show that "only if" part.

Suppose $x$ is of characteristic $O^{+}$. For every neighborhood $U$ of $K^{+}(x)$, there exists a neighborhood $V$ of $K^{+}(x)$ such that $\bar{V} \subset U$ and $\bar{V}$ is compact. If $W$ is 
a neighborhood of $x$ with $f\left(W \times \mathbb{R}^{+}\right) \subset V$, then $f(y) \subset \overline{f\left(W \times \mathbb{R}^{+}\right)} \subset \bar{V} \subset U$ for all $y \in W$. Hence $K^{+}$is upper semicontinuous at $x$. Consequently, $K^{+}$is continuous by Corollary 2.4. We next show the existence a neighborhood $W$ of $x$ with $f\left(W \times \mathbb{R}^{+}\right) \subset V$. Assume that this is not true, i.e., $f\left(W \times \mathbb{R}^{+}\right) \not \subset V$ for every neighborhood $W$ of $x$. Since $x \in K^{+}(x) \subset V, B(x, \epsilon) \subset V$ for some $\epsilon>0$. Also, by $f\left(B\left(x, \frac{\epsilon}{i}\right) \times \mathbb{R}^{+}\right) \not \subset V$ for all $i, f\left(x_{i}, t_{i}\right) \not \subset V$ for some $x_{i} \in B\left(x, \frac{\epsilon}{i}\right)$ and $t_{i} \in \mathbb{R}^{+}$. The connectivity of $f\left(x_{i},[0, t]\right)$ shows $f\left(x_{i},\left[0, t_{i}\right]\right) \cap \partial V \neq \emptyset$. Assume that $y_{i} \in f\left(x_{i},\left[0, t_{i}\right]\right) \cap \partial V$. Since $\partial V$ is compact, we may assume that $y_{i} \rightarrow y \in \partial V$. By $x_{i} \rightarrow x$ and $y_{i} \in f\left(x_{i},\left[0, t_{i}\right]\right) \subset f\left(x_{i}, \mathbb{R}^{+}\right), y \in D^{+}(x)=$ $K^{+}(x) \subset V$, which contradicts $y \notin V$. Hence there exists a neighborhood $W$ of $x$ with $f\left(W \times \mathbb{R}^{+}\right) \subset V$.

Definition 2.1. A subset $M \subset X$ is positively stable if for every neighborhood $U$ of $M$, there exists a neighborhood $V$ of $M$ such that $f\left(V \times \mathbb{R}^{+}\right) \subset U$.

Definition 2.2. A subset $M \subset X$ is positively invariant if $f\left(x, \mathbb{R}^{+}\right) \subset M$ for all $x \in M . M$ is positively minimal if $M$ is closed and positively invariant, but none of its nonempty proper subsets has these two properties.

Theorem 2.7. If $K^{+}$is upper semicontinuous on $K^{+}(x)$, then $K^{+}(x)$ is positively stable.

Proof. Let $U$ be any neighborhood of $K^{+}(x)$. By the upper semicontinuity of $K^{+}$on $K^{+}(x)$, for every point $y \in K^{+}(x)$, there exists a neighborhood $V_{y}$ of $y$ such that $z \in V_{y}$ implies $K^{+}(z) \subset U$. Since $f\left(z, \mathbb{R}^{+}\right) \subset \overline{f\left(z, \mathbb{R}^{+}\right)}=K^{+}(z) \subset$ $U$ for every $z \in V_{y}$, we get $f\left(V_{y} \times \mathbb{R}^{+}\right) \subset U$. Take $V=\bigcup_{y \in K^{+}(x)} V_{y}$. Then $V$ is a neighborhood of $K^{+}(x)$, and $f\left(V \times \mathbb{R}^{+}\right)=f\left(\bigcup_{y \in K^{+}(x)}\left(V_{y} \times \mathbb{R}^{+}\right)\right)$ $=\bigcup_{y \in K^{+}(x)} f\left(V_{y} \times \mathbb{R}^{+}\right) \subset U$.

Hence, $K^{+}(x)$ is positively stable.

Theorem 2.8. $K^{+}$is continuous if and only if $K^{+}(x)$ is positively stable for all $x \in X$.

Proof. Theorem 2.7 takes of the "only if" part. We now show that "if" part.

Suppose $K^{+}(x)$ is positively stable at every $x \in X$. For any neighborhood $U$ of $K^{+}(x)$, there exists a neighborhood $V$ of $K^{+}(x)$ such that $\bar{V} \subset U$. By the positive stability of $K^{+}(x), f\left(W \times \mathbb{R}^{+}\right) \subset V$ for some neighborhood $W$ of $K^{+}(x)$. This $W$ is a neighborhood of $x$. Hence, we get $K^{+}(y) \subset \overline{f\left(W \times \mathbb{R}^{+}\right)} \subset$ $\bar{V} \subset U$ for every $y \in W$. This shows that $K^{+}$is upper semicontinuous at $x$. By Corollary $2.4, K^{+}$is continuous at $x$.

Theorem 2.9. If $K^{+}(x)$ is positively stable at every $x \in X$, then so is $L^{+}(x)$.

Proof. We will show that $K^{+}(y)=L^{+}(x)$ for every $y \in L^{+}(x)$. Since $L^{+}(x)$ is positively invariant and compact, $L^{+}(x)$ contains a minimal set $M$. To prove that $M=L^{+}(x)$, assume that $y \in L^{+}(x)-M$. Then $y \in \bar{U}$ for some neighborhood $U$ of $M$. Let $z \in M$. We have $K^{+}(z)=M \subset U$. From 
the positive stability of $K^{+}(z), f\left(V \times \mathbb{R}^{+}\right) \subset U$ for some neighborhood $V$ of $K^{+}(z)$. Thus $f(x, t) \subset V$ for some $t \in \mathbb{R}^{+}$. As $f(x,[t, \infty))=f(f(x, t) \times$ $\left.\mathbb{R}^{+}\right) \subset f\left(V \times \mathbb{R}^{+}\right) \subset U, y \in L^{+}(x) \subset \overline{f(x,[t, \infty))} \subset \bar{U}$. This is impossible. Hence $M=L^{+}(x)$. Since $L^{+}(x)$ is a minimal set, $K^{+}(y)=L^{+}(x)$ for every $y \in L^{+}(x)$. By the minimality of $L^{+}(x), K^{+}(y)=L^{+}(x)$ for each $y \in L^{+}(x)$. By the assumption, $L^{+}(x)$ is positively stable.

Lemma 2.10. For any neighborhood $U$ of $L^{+}(x)$, there exists $t \in \mathbb{R}^{+}$such that $f(x,[t, \infty)) \subset U$.

Proof. By the local compactness of $X$, there exists a neighborhood $V$ of $L^{+}(x)$ such that $\bar{V} \subset U$ and $\bar{V}$ is compact. We will show that $f(x,[t, \infty)) \subset \bar{V}$ for some $t \in \mathbb{R}^{+}$. Assume that there exists $s \in \mathbb{R}^{+}$such that $s \geq t$ and $f(x, s) \not \subset \bar{V}$ for each $t \in \mathbb{R}^{+}$. Let $y \in L^{+}(x)$. By the definition of $L^{+}(x)$, there is a sequence $t_{n} \rightarrow+\infty$ with $y_{n} \in f\left(x, t_{n}\right)$ and $y_{n} \rightarrow y$. Since $V$ is a neighborhood of $y$, we may assume without loss of generality that $y_{n} \in V$ for all $n$. Hence $f\left(x, s_{n}\right) \not \subset \bar{V}$ for some $s_{n} \geq t_{n}$. By the connectivity of $f\left(x,\left[t_{n}, s_{n}\right]\right)$, we get $f\left(x,\left[t_{n}, s_{n}\right]\right) \cap \partial V \neq \emptyset$. Let $z_{n} \in f\left(x_{n}, r_{n}\right) \cap \partial V$ for $t_{n} \leq r_{n} \leq s_{n}$. As $\partial V$ is compact, $\left(z_{n}\right)$ has a convergent subsequence. Assume without loss of generality that $z_{n} \rightarrow z \in \partial V$. As $t_{n} \rightarrow \infty, y \in L^{+}(x)$. This is impossible. Consequently, $f(x,[t, \infty)) \subset \bar{V} \subset U$ for some $t \in \mathbb{R}^{+}$.

We now give a converse of Theorem 2.7.

Theorem 2.11. If $L^{+}(x)$ is positively stable, then so is $K^{+}(x)$.

Proof. Assume $K^{+}(x)$ is not positively stable. There exists a neighborhood $U$ of $K^{+}(x)$ such that $f\left(V \times \mathbb{R}^{+}\right) \not \subset U$ for every neighborhood $V$ of $K^{+}(x)$. By the local compactness of $X$, there is a neighborhood $W$ of $K^{+}(x)$ such that $\bar{W} \subset U$ and $\bar{W}$ is compact. Then, $B\left(K^{+}(x), \epsilon\right) \subset W$ for some $\epsilon>0$. As $f\left(B\left(K^{+}(x), \frac{\epsilon}{n}\right) \not \subset W\right.$ for all $n, f\left(B_{n}, \mathbb{R}^{+}\right) \not \subset W$ for some $x_{n} \in B\left(K^{+}(x), \frac{\epsilon}{n}\right)$. Since $x_{n} \in B\left(K^{+}(x), \frac{\epsilon}{n}\right) \subset W$ and $f\left(x_{n}, \mathbb{R}^{+}\right)$is connected set, we have

$$
f\left(x, \mathbb{R}^{+}\right) \cap \partial W \neq \emptyset .
$$

We may assume $y_{n} \in f\left(x_{n}, t_{n}\right) \cap \partial W$ for $t_{n} \in \mathbb{R}^{+}$. Since $\partial W$ is compact, we may assume $y_{n} \rightarrow y \in \partial W$. Also since $x_{n} \in \bar{W}$ and $\bar{W}$ is compact, we may assume $x_{n} \rightarrow z \in \bar{W}$. Clearly, $z \in K^{+}(x)$. If $\left(t_{n}\right)$ is bounded, we may assume $t_{n} \rightarrow t \in \mathbb{R}^{+}$. Since $\left(x_{n}, t_{n}\right) \rightarrow(z, t), y_{n} \in f\left(x_{n}, t_{n}\right)$ and the upper semicontinuity of $f$ at $(z, t), d\left(y_{n}, z_{n}\right) \rightarrow 0$ for some $z_{n} \in f(z, t)$. As $y_{n} \rightarrow y$, we get $z_{n} \rightarrow y$. Thus $y \in \overline{f(z, t)}=f(z, t) \subset K^{+}(x) \subset W$. This is impossible.

If $\left(t_{n}\right)$ is unbounded, we may assume $t_{n} \rightarrow \infty$. By the positive stability of $L^{+}(x), f\left(V \times \mathbb{R}^{+}\right) \subset W$ for some neighborhood $V$ of $L^{+}(x)$. Also, $f(x,[t, \infty)) \subset V$ for some $t \in \mathbb{R}^{+}$. Let $z \in f\left(x, \mathbb{R}^{+}\right) \cup L^{+}(x)$. If $z \in f\left(x, \mathbb{R}^{+}\right)$, then $z \in f(x, s)$ for some $s \in \mathbb{R}^{+}$. Thus $f(z, t) \subset f(f(x, s), t)=f(x, s+t) \subset$ $f(x,[t, \infty)) \subset V$. Since $f$ is upper semicontinuous at $(z, t), f(A, t) \subset V$ for some neighborhood $A$ of $z$. Choose $n$ such that $x_{n} \in A$ and $t_{n} \geq t$. 
Then $f\left(x_{n}, t_{n}\right) \subset f\left(x_{n},[t, \infty)\right)=f\left(f\left(x_{n}, t\right) \times \mathbb{R}^{+}\right) \subset f\left(f(A, t) \times \mathbb{R}^{+}\right) \subset$ $f\left(V \times \mathbb{R}^{+}\right) \subset W$. This is impossible. If $z \in L^{+}(x) \subset V, x_{n} \in V$ for some $n$. Thus, $f\left(x_{n}, \mathbb{R}^{+}\right) \subset f\left(V \times \mathbb{R}^{+}\right) \subset W$. Again this is impossible. Therefore $K^{+}(x)$ is positive stable.

Combining the above results, we have:

Theorem 2.12. The followings are equivalent.

(1) For each point $x \in X, x$ is of characteristic $O^{+}$.

(2) $K^{+}$is continuous.

(3) $K^{+}(x)$ is positively stable for each point $x \in X$.

(4) $L^{+}(x)$ is positively stable for each point $x \in X$.

\section{Continuity of the positive limit set map $L^{+}$, and its positive eventual stability}

Theorem 3.1. If the map $K^{+}$is upper semicontinuous at $x$, then so is the $\operatorname{map} L^{+}$.

Proof. Choose $x \in L^{+}(x)$. For every neighborhood $U$ of $L^{+}(x)=K^{+}(x)$, there exists a neighborhood $V$ of $x$ such that $y \in V$ implies $K^{+}(y) \subset U$. By $L^{+}(y) \subset$ $K^{+}(y)$, we get $L^{+}(y) \subset U$. Hence $L^{+}$is upper semicontinuous at $x$. Assume that $L^{+}$is not upper semicontinuous at $x \notin L^{+}(x)$. For some neighborhood $U$ of $L^{+}(x)$, there exists $y \in V$ such that $L^{+}(y) \not \subset U$ for every neighborhood $V$ of $x$. Since $K^{+}$is upper semicontinuous at $x, L^{+}(x)$ is positively stable by Lemma 2.4, Theorem 2.8 and Theorem 2.9. Thus, there exists positively invariant neighborhood $W$ of $L^{+}(x)$ such that $\bar{W} \subset U$ and $\bar{W}$ is compact. Also, $f(x,[s, \infty)) \subset W$ for some $s \in \mathbb{R}^{+}$. By the upper semicontinuity of $f$ at $(x, s), f(V, s) \subset W$ for some neighborhood $V$ of $x$. For each $n$, there exists $x_{n} \in B\left(x, \frac{1}{n}\right)$ such that $L^{+}\left(x_{n}\right) \not \subset U$. Since $x_{n} \rightarrow x$, we may assume that $x_{n} \in V$ for all $n$. We claim that $f\left(x_{n},[s, \infty)\right) \not \subset W$. Assume that $f\left(x_{n},[s, \infty)\right) \subset W$. Then, $L^{+}\left(x_{n}\right) \subset \overline{f\left(x_{n},[s, \infty)\right)} \subset \bar{W} \subset U$. This is impossible since $f\left(x_{n},[s, \infty)\right)=f\left(f\left(x_{n}, s\right) \times \mathbb{R}^{+}\right) \subset f\left(f(V, s) \times \mathbb{R}^{+}\right) \subset$ $f\left(W \times R^{+}\right)=W$. Thus $f\left(x_{n},[s, \infty)\right) \not \subset W$.

On the other hand, by $f\left(x_{n}, s\right) \subset f(V, s) \subset W$ and the connectedness of $f\left(x_{n},[s, \infty)\right)$, we get $f\left(x_{n},[s, \infty)\right) \cap \partial W \neq \emptyset$. Setting $y_{n} \in f\left(x_{n}, t_{n}\right) \cap \partial W$ for $t_{n} \geq s$. By compactness of $\partial W$, we may assume that $y_{n} \rightarrow y \in \partial W$. If $\left(t_{n}\right)$ is bounded, assume without loss of the generality that $t_{n} \rightarrow t$ for $t \geq s$. From $\left(x_{n}, t_{n}\right) \rightarrow(x, t), y_{n} \in f\left(x_{n}, t_{n}\right)$ and upper semicontinuity of $f$ at $(x, t)$, we get $d\left(y_{n}, z_{n}\right) \rightarrow 0$ for some $z_{n} \in f(x, t)$. Also, $z_{n} \rightarrow y$ since $y_{n} \rightarrow y$. Thus, $y \in \overline{f(x, t)}=f(x, t) \subset f(V \times[s, \infty)) \subset W$. This is impossible. If $\left(t_{n}\right)$ is unbounded, we may assume without loss of the generality $t_{n} \rightarrow \infty$. By Lemma 2.4, $x$ is of characteristic $O^{+}$. Thus, $y \in J^{+}(x)=L^{+}(x) \subset W$. This is impossible.

Consequently, $L^{+}$is upper semicontinuous at $x$. 
Definition 3.1. A set $M \subset X$ is positively eventually stable if for every neighborhood $U$ of $M$, there exists a neighborhood $V$ of $M$ such that for every $x \in V$, there exists $t \in \mathbb{R}^{+}$such that $f(x,[t, \infty)) \subset U$.

An important concept in the theory of general dynamical systems is that of a motion.

Definition 3.2. A continuous function $\Phi: \mathbb{R} \rightarrow X$ is called a motion of $f$ if $\Phi(t) \in f(\Phi(s), t-s)$ for $s<t$.

Let $\Psi$ be the set of all motions of $f$. For $x \in X$, let $\Psi(x)=\{\Phi \in$ $\Psi \mid \Phi(0)=x\}$. Let $\Phi \in \Psi$. The positivie limit set $L^{+}(\Phi)$ of $\Phi$ is defined as $L^{+}(\Phi)=\bigcap_{t>0} \overline{\Phi([t, \infty))}$.

Lemma 3.2. Let $\Phi \in \Psi$. Then $x \in L^{+}(\Phi)$ if and only if there exists a sequence $\left(t_{n}\right)$ in $\mathbb{R}$ with $t_{n} \rightarrow+\infty$ and $\Phi\left(t_{n}\right) \rightarrow x$.

Proof. The proof easily follows from the definition.

Lemma 3.3. Let $\Phi \in \Psi$. Then for all $s \in \mathbb{R}, L^{+}(\Phi) \subset L^{+}(\Phi(s))$.

Proof. Let $y \in L^{+}(\Phi)$. Then there exists a sequence $\left(t_{n}\right)$ in $\mathbb{R}$ with $t_{n} \rightarrow+\infty$ and $\Phi\left(t_{n}\right) \rightarrow y$. We may assume without loss of the generality $t_{n}>s$ for all $n$. Since $\Phi\left(t_{n}\right) \in f\left(\Phi(s), t_{n}-s\right)$ and $t_{n}-s \rightarrow \infty$, we get $y \in L^{+}(\Phi(s))$. This proves the lemma.

We assume that $f$ has the following assumption.

If $y \in f(x, s)$ and $z \in f(x, t)$ for $s<t$, then there exists $\Phi \in \Psi(y)$ such that $\Phi(t-s)=z$.

Now, under the hypothesis, we will prove the following results in Section 3.

Lemma 3.4. For all $y \in L^{+}(x), y \in L^{+}(\Phi)$ for some $\Phi \in \Psi(x)$.

Proof. By the definition of $L^{+}(x)$, there exist sequences $\left(t_{n}\right)$ in $\mathbb{R}$ and $\left(y_{n}\right)$ in $f\left(x, t_{n}\right)$ with $t_{n} \rightarrow+\infty, y_{n} \rightarrow y$. We may assume that $t_{1}<t_{2}<t_{3}<\cdots$. Then, there are $\phi_{1} \in \Psi(x)$ and $\Phi \in \Psi\left(y_{1}\right)$ such that $\phi_{1}\left(t_{2}-t_{1}\right)=y_{2}, \Psi\left(t_{2}-t_{1}\right)=$ $y_{2}$. We define a map $\Phi_{2}: \mathbb{R} \rightarrow X$ by

$$
\Phi_{2}(t)= \begin{cases}\phi_{1}(t) & \text { if } t \leq t_{1} \\ \Phi_{1}\left(t-t_{1}\right) & \text { if } t \geq t_{1}\end{cases}
$$

Then, $\Phi_{2}$ is continuous and $\Phi_{2}\left(t_{2}\right)=y_{2}$. Let $s<t$. Consider the following three cases;

Case 1. $t_{1}<s$

$\Phi_{2}(t)=\Phi_{1}\left(t-t_{1}\right) \in f\left(\Phi_{1}\left(s-t_{1}\right), t-s\right)=f\left(\Phi_{2}, t-s\right)$.

Case 2. $s \leq t_{1}<t$

$\Phi_{2}(t)=\Psi\left(t-t_{1}\right) \in f\left(\Psi(0), t-t_{1}\right)=f\left(y_{1}, t-t_{1}\right)=f\left(\Phi_{1}\left(t_{1}\right), t-t_{1}\right) \subset$ $f\left(f\left(\Phi_{1}(s), t_{1}-s\right), t-t_{1}\right)=f\left(\Phi_{1}(s), t-s\right)=f\left(\Phi_{2}(s), t-s\right)$.

Case 3. $t \leq t_{1}$

$\Phi_{2}(t)=\Phi_{1}(t) \in f\left(\Phi_{1}(s), t-s\right)=f\left(\Phi_{2}(s), t-s\right)$. 
Thus, we obtain $\Phi_{2} \in \Psi(x), \Phi_{2}=\Phi_{1}$ on $\left(-\infty, t_{1}\right]$. Proceeding in this fashion, we obtain $\Phi_{n} \in \Psi(x)$ with the property that $\Phi_{n}\left(t_{n}\right)=y_{n}$ and $\Phi_{n}=$ $\Phi_{n-1}$ on $\left(-\infty, t_{n-1}\right]$.

We define a function $\Phi: \mathbb{R} \rightarrow X$ by $\Phi(t)=\Phi_{n}(t)$ for some $n$ with $t_{n}>t$. Then since $\Phi=\Phi_{n}$ on $\left(-\infty, t_{n}\right]$, it follows that $\Phi \in \Psi(x)$ and $\Phi\left(t_{n}\right)=y_{n}$ for all $n$.

Consequently $y \in L^{+}(\Phi)$. The lemma is proved.

Theorem 3.5. Given $x \in X, L^{+}$is upper semicontinuous on $L^{+}(x)$ if and only if $L^{+}(x)$ is positively minimal and positively eventually stable.

Proof. Suppose $L^{+}$is upper semicontinuous. Let $y \in L^{+}(x)$. We shall show $L^{+}(x) \subset L^{+}(y)$. Assume $z \in L^{+}(x)-L^{+}(y)$. Then $z \notin U$ for some neighborhood $U$ of $L^{+}(y)$. As upper semicontinuity of $L^{+}$at $y$, there exists a neighborhood $V$ of $y$ such that $p \in V$ implies $L^{+}(p) \subset U$. Also, there exist sequences $s_{n} \rightarrow \infty, t_{n} \rightarrow \infty, y_{n} \in f\left(x, s_{n}\right), z_{n} \in f\left(x, t_{n}\right)$ such that $y_{n} \rightarrow y$, $z_{n} \rightarrow z$. We may assume without loss of the generality $s_{1}<t_{1}<s_{2}<$ $t_{2}<\cdots<s_{n}<t_{n}<\cdots$. By similar method of proof of Lemma 3.4, there exists $\Phi \in \Psi(x)$ such that $\Phi\left(s_{n}\right)=y_{n}, \Phi\left(y_{n}\right)=z_{n}$. Thus, we get $y, z \in L^{+}(\Phi)$ for some $\Phi \in \Psi(x)$. As $y \in L^{+}(\Phi)$, it follows that $\Phi(t) \in V$ for some $t \in \mathbb{R}^{+}$. Thus, $z \in L^{+}(\Phi) \subset L^{+}(\Phi(t)) \subset U$. This is absurd as $z \notin U$. So $L^{+}(x)$ is positively minimal. We now prove that $L^{+}(x)$ is positively eventually stable. Let $U$ be a neighborhood of $L^{+}(x)$. By upper semicontinuity of $L^{+}$at $y \in L^{+}(x)$, there exists neighborhood $V_{y}$ of $y$ such that $z \in V_{y}$ implies $L^{+}(z) \subset U$. Setting $V=\bigcup_{y \in L^{+}(x)} V_{y}$. Then $V$ is a neighborhood of $L^{+}(x)$. Also, for all $z \in V$, there exists $y \in L^{+}(x)$ such that $z \in V_{y}$. Thus, since $L^{+}(z) \subset U$, by Lemma 2.10 , there exists $t \in \mathbb{R}^{+}$such that $f(z,[t, \infty)) \subset U$. This shows $L^{+}(x)$ is positively eventually stable.

Conversely let $L^{+}(x)$ be positively minimal and positively eventually stable. Let $y \in L^{+}(x)$ and let $U$ be a neighborhood of $L^{+}(y)$. By positive minimality of $L^{+}(x), L^{+}(x)=L^{+}(y)$. There exists a neighborhood $V$ of $L^{+}(x)$ such that $\bar{V} \subset$ $U$. Since $L^{+}(x)$ is positively eventually stable, there exists a neighborhood $W$ of $L^{+}(x)$ such that for every $z \in W$, there exists $t \in \mathbb{R}^{+}$such that $f(z,[t, \infty)) \subset$ $U$. Thus, $L^{+}(z) \subset \overline{f(z,[t, \infty))} \subset \bar{V} \subset U$. Hence $L^{+}$is upper semicontinuous on $L^{+}(x)$.

Definition 3.3. With a given $M \subset X$,

$$
A(M)=\left\{x \in X \mid L^{+}(x) \neq \emptyset \text { and } L^{+}(x) \subset M\right\} .
$$

This set called the region of attraction of the set $M$.

Lemma 3.6. The map $L^{+}$is upper semicontinuous on $L^{+}(x)$ if and only if $L^{+}$is upper semicontinuous on $A\left(L^{+}(x)\right)$.

Proof. As $L^{+}(x) \subset A\left(L^{+}(x)\right)$, we only need prove that $L^{+}$is upper semicontinuous on $A\left(L^{+}(x)\right)$, if $L^{+}$is upper semicontinuous on $L^{+}(x)$. So let 
$y \in A\left(L^{+}(x)\right)$ and let $U$ be a neighborhood of $L^{+}(y)$. There exists a neighborhood $V$ of $L^{+}(y)$ such that $\bar{V} \subset U$. As $L^{+}(x)$ is positively minimal, $L^{+}(x)=L^{+}(y)$ by Theorem 3.5. As $L^{+}(x)$ positively eventually stable, there exists a neighborhood $W$ of $L^{+}(y)$ such that for each $z \in W$, there exists $t \in \mathbb{R}^{+}$such that $f(z,[t, \infty)) \subset V$. Also, $f(y,[s, \infty)) \subset W$ for some $s \in \mathbb{R}^{+}$. Since $f$ is upper semicontinuous at $(y, s)$, there exists a neighborhood $A$ of $y$ such that $f(A, s) \subset W$. So, for every $p \in W$, there exists $t \in \mathbb{R}^{+}$such that $f(f(p, s),[t, \infty))=f(p,[s+t, \infty)) \subset V$. Thus, $L^{+}(p) \subset \overline{f(p,[s+t, \infty))} \subset \bar{V} \subset U$. Hence $L^{+}$is upper semicontinuous on $A\left(L^{+}(x)\right)$.

Lemma 3.7. If $L^{+}(y)$ is positively eventually stalbe for each $y \in L^{+}(x)$, then $L^{+}(x)$ is positively minimal.

Proof. Suppose $L^{+}(x)$ is not positively minimal. Then there exists a minimal set $M$ in $L^{+}(x)$. Let $y \in L^{+}(x)-M$. There exists a neighborhood $U$ of $M$ such that $x \notin \bar{U}$. Let $z \in M$. By assumption, $L^{+}(x)=M$ is positively eventually stable. Therefore, there exists a neighborhood $V$ of $M$ such that for every $p \in V$, there exists $t \in \mathbb{R}^{+}$such that $f(p,[t, \infty)) \subset U$. There exists $\Phi \in \Psi(x)$ such that $y, z \in L^{+}(\Phi)$. Since $V$ is a neighborhood of $z$, $\Phi(s) \in V$ for some $s \in \mathbb{R}^{+}$. So that $f(\Phi(s),[t, \infty)) \subset U$ for some $t \in \mathbb{R}^{+}$. Hence $y \in L^{+}(\Phi) \subset L^{+}(\Phi(s)) \subset \overline{f(\Phi(s),[t, \infty))} \subset \bar{U}$. This is impossible since $y \notin \bar{U}$. Thus $L^{+}(x)$ must be positively minimal.

Combining Theorem 3.5, Lemma 3.6 and Lemma 3.7 we have:

Theorem 3.8. The map $L^{+}$is upper semicontinuous on $X$ if and only if $L^{+}(x)$ is positively eventually stable for each $x \in X$.

Proof. Since $L^{+}(x)$ is positively eventually stable for each $x \in X$, by Lemma $3.7, L^{+}(x)$ is positively minimal. Also by Theorem $3.5, L^{+}$is upper semicontinuous. According to Lemma 3.6, $L^{+}$is upper semicontinuous on $A\left(L^{+}(x)\right)$. Since $x \in A\left(L^{+}(x)\right), L^{+}$is upper semicontinuous for each $x \in X$.

Conversely, the proof is immediate by Theorem 3.5 .

We now introduce the concept of positively eventually weakly stable which shows the connection between the continuity of $L^{+}$and positively eventually weak stability of $L^{+}(x)$ for each $x \in X$.

Definition 3.4. A set $M \subset X$ is positively eventually weakly stable if for every neighborhood $U$ of $M$, there exists a neighborhood $V$ of $M$ such that $U \cap f(x,[t, \infty)) \neq \emptyset$ for each $x \in V, t \in \mathbb{R}^{+}$.

Lemma 3.9. Let $L^{+}(y)$ be positively minimal for each $y \in L^{+}(x)$. Then the map $L^{+}$is lower semicontinuous on $L^{+}(x)$ if and only if $L^{+}(y)$ is positively eventually weakly stable for every $y \in L^{+}(x)$. 
Proof. Let $y \in L^{+}(x)$ for each $x \in X$ and let $U$ be a neighborhood of $L^{+}(y)$. For all $z \in L^{+}(y)$, we obtain $L^{+}(z) \cap U \neq \emptyset$. By lower semicontinuity of $L^{+}$at $z$, there exists neighborhood $V_{z}$ of $z$ such that $w \in V_{z}$ implies $L^{+}(w) \cap U \neq \emptyset$.

Setting $V \equiv \bigcup_{z \in L^{+}(y)} V_{z}$. Then, $V_{z}$ is a neighborhood of $L^{+}(y)$. For each $w \in V, w \in V_{z}$ for some $z \in L^{+}(y)$. Since $L^{+}(w) \cap U \neq \emptyset$, we obtain $U \cap f(w,[t, \infty)) \neq \emptyset$ for all $t \in \mathbb{R}^{+}$. Hence $L^{+}(y)$ is positively eventually weakly stable for every $y \in L^{+}(x)$.

Conversely, let $y \in L^{+}(x)$ and $U$ be open set in $X$ with $L^{+}(y) \cap U \neq \emptyset$. Positive minimality of $L^{+}(y)$ ensures that $K^{+}(z) \cap U \neq \emptyset$ for all $z \in L^{+}(y)$. By lower semicontinuity of $K^{+}$at $z$, there exists a neighborhood $V_{z}$ of $z$ such that $w \in V_{z}$ implies $K^{+}(w) \cap U \neq \emptyset$. Also there exists a neighborhood $W_{z}$ such that $\overline{W_{z}} \subset V_{z}$ and $\overline{W_{z}}$ is compact. Consider an open cover $\left\{W_{z} \mid z \in L^{+}(y)\right\}$ of $L^{+}(y)$. By compactness of $L^{+}(y)$, there are finite points $z_{1}, z_{2}, \ldots, z_{m}$ in $L^{+}(y)$ such that $L^{+}(y) \subset \bigcup_{i=1}^{m} W_{z_{i}}$.

Setting $W=\bigcup_{i=1}^{m} W_{z_{i}}$. Then $W$ is a neighborhood of $L^{+}(y)$. As $L^{+}(y)$ is positively eventually weakly stable, there exists a neighborhood $A$ of $L^{+}(y)$ such that $W \cap f(w,[t, \infty)) \neq \emptyset$ for every $w \in A, t \in \mathbb{R}^{+}$. Also, there exists a $s \in \mathbb{R}^{+}$such that $f(y, s) \subset A$. By the upper semicontinuity of $f$ at $(y, s)$ there exists a neighborhood $B$ of $y$ such that $f(B, s) \subset A$. Let $p \in f(b, s)$ for all $b \in B$. Since $p \in f(b, s) \subset f(B, s) \subset A$, we get $W \cap f(p,[n, \infty)) \neq \emptyset$ for $n$. As $f(p,[n, \infty)) \subset f(f(b, s),[n, \infty))=f(b,[n+s, \infty))$, we obtain $W \cap f(b,[n+$ $s, \infty)) \neq \emptyset$. Let $x_{n} \in W \cap f\left(b, t_{n}\right)$ for $t_{n} \geq n+s$. By compactness of $\bar{W}$ and $x_{n} \in W \subset \bar{W}$, we may assume $x_{n} \rightarrow w \in \bar{W}$. Since $x_{n} \in f\left(b, t_{n}\right), t_{n} \rightarrow \infty$, we have $w \in L^{+}(b)$. According to $\bar{W}=\overline{\bigcup_{i=1}^{n} W_{z_{i}}}=\bigcup_{i=1}^{n} \overline{W_{z_{i}}} \subset \bigcup_{i=1}^{n} V_{z_{i}}$, we obtain $w \in V_{z_{i}}$ for some $i$. Hence $K^{+}(w) \cap U \neq \emptyset$. By $w \in L^{+}(b)$, we get $K^{+}(w) \subset L^{+}(b)$. Therefore $L^{+}(b) \cap U \neq \emptyset$. Thus $L^{+}$is lower semicontinuous on $L^{+}(x)$.

Corollary 3.10. If the map $L^{+}$is upper semicontinuous on $L^{+}(x)$, then $L^{+}$ is lower semicontinuous on $L^{+}(x)$.

Proof. Since $L^{+}(y) \subset L^{+}(x)$ for all $y \in L^{+}(x), L^{+}$is upper semicontinuous on $L^{+}(y)$. By Theorem $3.5, L^{+}(y)$ is positively minimal and positively eventually stable. Thus $L^{+}$is lower semicontinuous on $L^{+}(x)$ by Lemma 3.9.

Theorem 3.11. A necessary and sufficient condition that the map $L^{+}$be continuous on $L^{+}(x)$ is that $L^{+}(x)$ is positively minimal and positively eventually stable.

Proof. If $L^{+}$is continuous on $L^{+}(x)$, by Theorem $3.4, L^{+}(x)$ is positively minimal and positively eventually stable.

Conversely, the proof is immediate from Theorem 3.4 and Corollary 3.10.

Theorem 3.12. A necessary and sufficient condition that the map $L^{+}$be continuous on $X$ is that $L^{+}(x)$ is positively eventually stable for each $x \in X$. 
Proof. Suppose $L^{+}(x)$ is positively eventually stable for each $x \in X$. By Theorem 3.8 and Corollary 3.10, $L^{+}$is lower semicontinuous on $L^{+}(x)$. If $L^{+}$is lower semicontinuous on $A\left(L^{+}(x)\right)$, then $L^{+}$is lower semicontinuous on $x \in X$. So we show the lower semicontinuity of $L^{+}$on $A\left(L^{+}(x)\right)$. Let $y \in A\left(L^{+}(x)\right)$. Then $L^{+}(y) \subset L^{+}(x)$. By Theorem 3.5 and Theorem 3.8, $L^{+}(x)$ is positively minimal. So, $L^{+}(y)=L^{+}(x)$. Let $U$ be an open set in $X$ with $U \cap L^{+}(y) \neq \emptyset$. Then for all $z \in L^{+}(y), L^{+}(z) \cap U \neq \emptyset$. By the lower semicontinuity of $L^{+}$at $z$, there exists a neighborhood $V_{z}$ of $z$ such that $w \in V_{z}$ implies $L^{+}(w) \cap U \neq \emptyset$.

Setting $V=\bigcup_{z \in L^{+}(y)} V_{z}$. Then $V$ is a neighborhood of $L^{+}(y)$. Hence $f(y,[t, \infty)) \subset V$ for some $t \in \mathbb{R}^{+}$. By the upper semicontinuity of $f$ at $(y, t)$, there exists a neighborhood $W$ of $y$ such that $f(W, t) \subset V$. Choose a point $q$ in $f(p, t)$ for all $p \in W$. Since $q \in f(p, t) \subset f(W, z) \subset V$, we obtain $q \in V_{z}$ for some $z \in L^{+}(y)$. As $L^{+}(q) \cap U \neq \emptyset$ and $L^{+}(q) \subset L^{+}(p)$, we get $L^{+}(p) \cap U \neq \emptyset$. Hence $L^{+}$is lower semicontinuous at $y$. Therefore, $L^{+}$is lower semicontinuous on $A\left(L^{+}(x)\right)$.

\section{References}

[1] N. P. Bhatia and G. P. Szego, Stability Theory of Dynamical Systems, Springer Verlag, New York, 1970.

[2] S. Elaydi and S. K. Kaul, Asymptotic stability in general dynamical systems, Nonlinear Anal. 13 (1989), no. 6, 657-669.

[3] D. Li, Morse decompositions for general dynamical systems and differential inclusions with applications to control systems, SIAM J. Control Optim. 46 (2007), no. 1, 35-60.

[4] $\longrightarrow$, On dynamical stability in general dynamical systems, J. Math. Anal. Appl. 263 (2001), no. 2, 455-478.

[5] E. Roxin, Stability in general control system, J. Differential Equations 1 (1965), 115-150.

[6] S. H. Saperstone and M. Nishihama, Continuity of the limit set maps in semidynamical systems, J. Differential Equations 23 (1977), no. 2, 183-199.

[7] K. S. Sibirsky, Introduction to Topological Dynamics, Noordhoff international Pub. Leyden, 1975.

KYung BoK LeE

Department of Mathematics

HoSEO UNIVERSity

Chungnam 337-850, Korea

E-mail address: kblee@hoseo.edu

JoNG SuH PARK

Department of Mathematics

Chungnam National University

TAEJON 305-764, KoreA

E-mail address: jspark@math.cnu.ac.kr 